\title{
Interdisciplinary shared governance: A literature review
}

\author{
Salwa Alrwaihi ${ }^{1}$, V. Kehyayan ${ }^{2}$, Jessie M. Johnson*2 \\ ${ }^{1}$ Hamad Medical Corporation, Doha, Qatar \\ ${ }^{2}$ School of Nursing, University of Calgary Qatar, Doha, Qatar
}

Received: November 1, 2017

Accepted: November 26, $2017 \quad$ Online Published: November 28, 2017

DOI: $10.5430 /$ jnep.v8n4p43

URL: https://doi.org/10.5430/jnep.v8n4p43

\begin{abstract}
Objective: Interdisciplinary shared governance (IDSG) is important in healthcare to achieve quality and excellence in patient care. Initially adopted in healthcare facilities for the nursing discipline, the recent trend is to expand it to include other disciplines. This paper examined the factors that affect interdisciplinary collaboration that effect successful implementation of an IDSG model.

Methods: A literature review on SG, interdisciplinary collaboration, and factors that may potentially influence its successful implementation was conducted.

Results: The review of the literature identified several factors grouped under three major themes that affect IDSG. The first theme was individual factors that include the subthemes provider attitude, beliefs, interpersonal skills, and status quo. The second theme was shared factors that includes both individual as well as organizational factors. They include physician-nurse relationships, clear goals and vision, motivation, trust and respect, and team functional skills. Finally, the organizational factors refer to those that impact the working environment and influence decision making by members of the various interdisciplinary teams. These factors include organizational structure, organizational culture, leadership, education, resources, professional boundary, and role ambiguity.

Conclusions: The IDSG requires that groups in different disciplines make informed decisions pertaining their work environment as well as those towards patient's care.
\end{abstract}

Key Words: Shared governance, Interdisciplinary, Interprofessional, Multidisciplinary health care, Interdisciplinary collaboration

\section{INTRODUCTION}

Health care systems today are in major global transformation to give effective, efficient, safe, evidence-based, and collaborative care to achieve excellence in patient care. ${ }^{[1]}$ Shared governance (SG) is a major innovation and evidence-based method to empower staff nurses in making decisions that affect their work environment and practice. ${ }^{[2]}$ The introduction of the SG as an organizational structure model was due to the severe shortage and high turnover of nurses in the past decades. The introduction of SG assisted in improving nurses' working conditions and helped healthcare organizations to provide the best health care available thus benefiting the nurses, the stakeholders, the patients as well as the health care organizations itself. The SG started with the nursing profession because most points of service staff are nurses and if the change process went smoothly with nurses, the transfer to interdisciplinary shared governance will be easy.

*Correspondence: Jessie M. Johnson; Email: Jessie.johnson1@ucalgary.ca; Address: School of Nursing, University of Calgary Qatar, Doha, Qatar. 
The quality of patient care involves the combined effort of all disciplines working in any health care organization. All disciplines shared a common goal that is to deliver the best care with minimum risk to patient safety. So, it is very important to work in collaboration with other disciplines to ensure that the patients receive the best care. The interdisciplinary shared governance (IDSG) is defined as different disciplines working together to make informed decisions pertaining their work environment as well as those towards patients' care. ${ }^{[3]}$

\section{METHODS}

The method used for this paper was a literature review. A literature review is defined as the systematic identification, evaluation, and interpretation of different works produced by various scholars, practitioners, and researchers. ${ }^{[4]}$ This approach was chosen to provide a broad overview of the IDSG model, which is a relatively new model in healthcare organizations. This paper synthesizes available information on IDSG models, the factors that potentially influence their successful implementation, and the factors that affect inter- disciplinary collaboration.

A comprehensive literature search of CINAHL and Medline databases were used to identify relevant articles using Keywords such as Shared Governance, Interdisciplinary, Interprofessional, Multidisciplinary Health Care, Organizational Factors, Interdisciplinary Collaboration were used. All English language papers that described the development and implementation of SG models were included. No timeframes were specified to include interdisciplinary collaboration from the inception of the concept to the present (2016). Eligible articles were not limited to empirical research but included theoretical papers, discussions, and case studies to obtain all SG models and interdisciplinary collaboration published to 2016. The retrieved articles were assessed for their eligibility based on the inclusion criteria such as English language, open timeframe, empirical research, discussion articles and case studies containing information related to SG, interdisciplinary collaboration, and IDSG. Articles were excluded if they were in a language other than English or contained literature not related to SG or interdisciplinary collaboration.

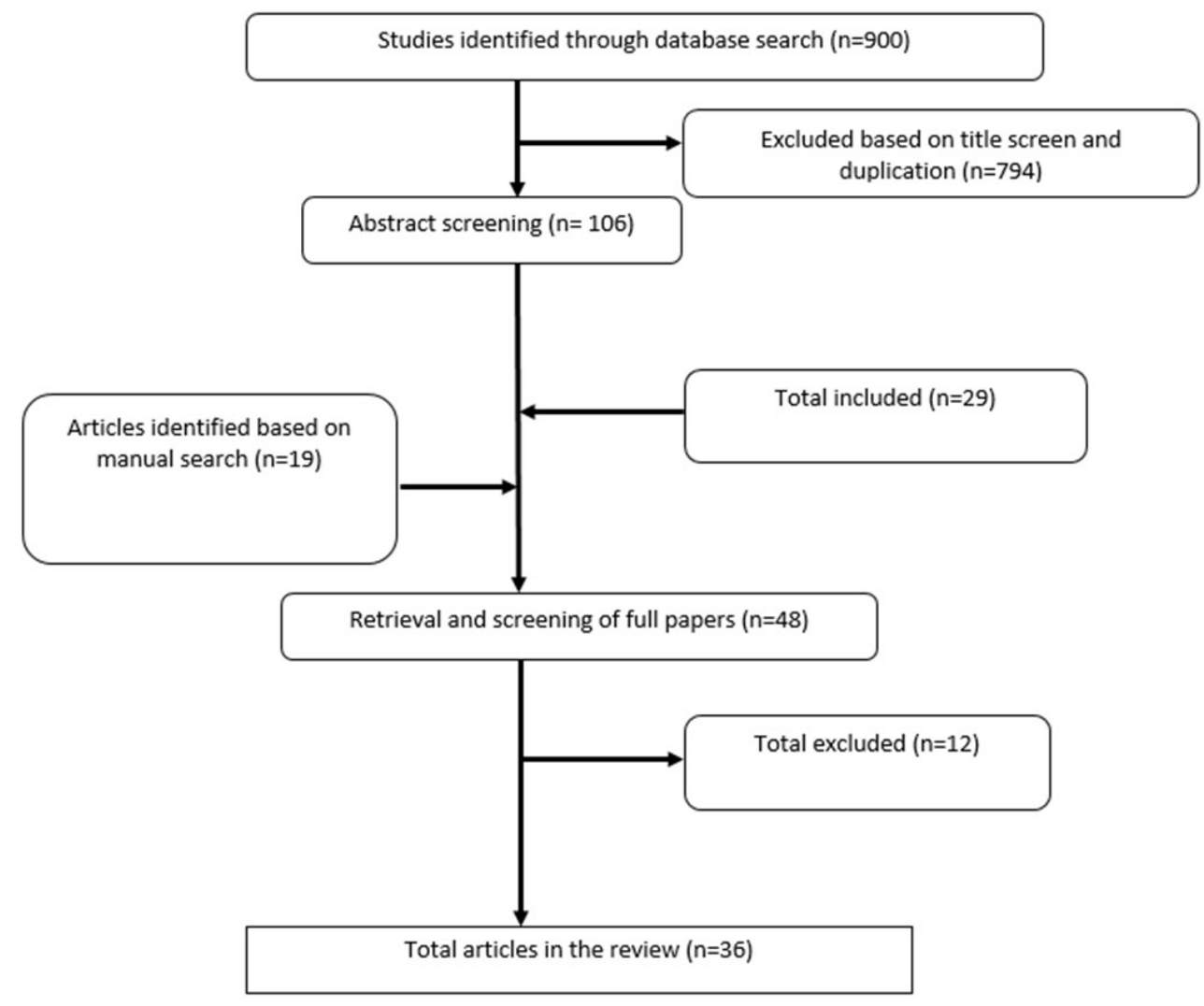

Figure 1. Flow diagram showing search strategy and results
3. FACTORS INFLUENCING INTERDISCI- categorized into three major themes: individual factors, other

\section{PLINARY COLLABORATION}
This literature review identified several barriers and facili- tators of interdisciplinary collaboration. These factors are factors, and organizational factors. Within each of these ma- jor themes, subthemes are also identified and discussed (see Figure 2). 44 


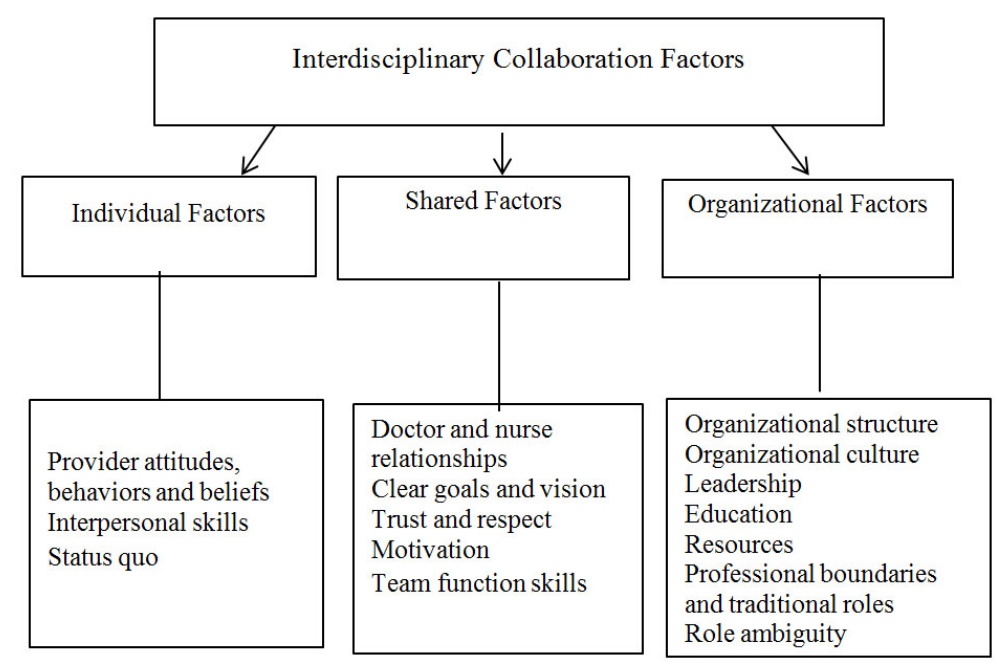

Figure 2. Interdisciplinary collaboration factors

\subsection{Individual factors}

The literature review identified three subthemes under individual factors that affect interdisciplinary collaboration. These individual factors are provider attitude, behavior and belief, interpersonal skills, and status quo.

\subsubsection{Provider attitudes, behaviors, and beliefs}

Attitudes, individual behaviors, and beliefs affect the collaboration of interdisciplinary professionals in SG. ${ }^{[5-9]}$ A positive belief in the importance of collaboration increases the success of the collaborative process. A positive attitude leads to the development of a respectful and welcoming attitude among different members of the interdisciplinary team. However, negative attitudes hinder the development of a positive working environment that will encourage interdisciplinary collaboration. The behaviors of interdisciplinary team members are influenced by the beliefs held by them. A negative attitude will also lead to lower commitment towards meeting shared healthcare goals. Personal beliefs that result from the members' past socialization processes and cultural experiences may generate superiority or inferiority complex. A belief that nurses are less experienced or knowledgeable, when compared to doctors, will for instance, hinder effective collaboration in the SG process. Health professionals, for instance, consider professionals from other disciplines as less knowledgeable when dealing with issues relating to health. ${ }^{[10,11]}$ Noted that beliefs and attitudes were central to the elimination or creation of barriers in teams.

According to Pullon's study, ${ }^{[12]}$ the behavior of individuals affects the respect and trust among team members which in turn will affect the success of IDSG. Such teams are usually made up of individuals with different personalities which influence success levels of SG for promoting the quality of healthcare. Individuals with a professional attitude are likely to develop and share governance more respectfully in the process of delivering high quality of health. ${ }^{[13]}$

The behaviors and beliefs of individuals in an interdisciplinary team play an important role in ensuring effective SG for the identification of problems and innovative ideas aimed at improving the quality of healthcare provided. ${ }^{[14]}$

\subsubsection{Interpersonal skills}

Interpersonal skills form the basis for engagement in a SG process that involves interdisciplinary members. Interpersonal skills refer to skills that members of the interdisciplinary team have and will utilize for effective interaction. Lack of interpersonal skills will inhibit the ability of the interdisciplinary team members to interact effectively in an SG model. Good interpersonal skills are a prerequisite to the development of a good rapport among interdisciplinary members. Positive interpersonal skills involve the ability to communicate effectively with respect and dignity irrespective of the position an individual may have on the team. Good interpersonal skills help create a good rapport among members of the team which eventually helps develop a bond among the interdisciplinary team members. Since the interdisciplinary team members share governance and ideas, it is necessary that team members are receptive and can work with individuals having different personalities and perspectives. ${ }^{[15]}$ Interdisciplinary collaboration depends on positive interpersonal skills that can lead to the development of a healthy work environment in the process of providing high-quality care. ${ }^{[16]}$ Giving nurses an opportunity to improve their interpersonal skills may help in improving decision-making in the shared governance process. ${ }^{[11,17-21]}$ Self-awareness is key to improving communication skills and better sharing of information among interdisciplinary teams. ${ }^{[22]}$ 


\subsubsection{Status quo}

The status quo represents a very common barrier among members not willing to accommodate new ideas. ${ }^{[17,23]}$ Some nurses and physicians refuse to acknowledge or recognize the need for a new structure and partnership. ${ }^{[24]}$ There can also be difficulties among staff with regards to identifying with new roles in an SG model; for instance, nurses and other health care professionals are conditioned both socially and educationally to accept hierarchical authority even in the context of SG. ${ }^{[8]}$ Management needs to engage staff in the change process to reduce staff resistant. ${ }^{[23]}$ A key driving force for the successful implementation of an IDSG was professionals who were dissatisfied with the status quo ${ }^{[11]}$ and hence could serve as advocates and champions for the successful implementation of IDSG.

\subsection{Shared factors}

This literature review identified several subtheme factors that may be considered both as individual and organizational factors. These factors are physician and nurse relationships, clear goals and vision, trust and respect, motivation and team function skills.

\subsubsection{Doctor and nurse relationships}

According to Pullo ${ }^{[12]}$ argued that problematic relationships between nurses and doctors are likely to limit collaboration. His studies focused on understanding the impact of attitudes and perceptions between physicians and nurses on SG. Negative attitudes between nurses and physicians may arise due to personal and relational attitudes during healthcare delivery which negatively affects IDSG. Porter-O'Grady ${ }^{[8]}$ noted that disputes between nurses emerge over the roles each should play. It is through effective communication that such disputes are resolved. Whereas physicians tend to negotiate with nurses, nurses are likely to avoid communication in cases where conflicts arise.

\subsubsection{Clear goals and vision}

A clear understanding of common goals, as well as a clear vision, constitutes an important factor in the success of collaboration among healthcare professionals. By focusing on the common goal, without distinction of titles, functions, or tasks, a "boundary-less" interdisciplinary partnership may be formed. ${ }^{[15,19-21,24]}$ According to George et al.'s study, ${ }^{[25]}$ objectivity and the development of clear goals by a team enhances the process of collaboration. The team members, through interdisciplinary collaboration, share a vision and can function more effectively. ${ }^{[26]}$ The contribution of every member of the interdisciplinary team should be respected. ${ }^{[21,27-29]}$

\subsubsection{Trust and respect}

Effective collaboration among members of an interdisciplinary team relies on respect and mutual trust. ${ }^{[12,13,15,22,28,30-32]}$ The loss of trust in a team breeds skepticism among its members. Mistrust impacts negatively on interpersonal relationships making it challenging for team members to work together. ${ }^{[22]}$ Confidence is reduced significantly with reduced mistrust among members of an interdisciplinary team. Trust or mistrust within an organization forms the basis on which relationships are built and therefore affects how open members of an interdisciplinary team become in IDSG. However, members of a team cultivate trust by working together over time. Mahdizadeh et al. ${ }^{[32]}$ noted that trust among nurses and physicians at different organizational levels are important in the creation of a professional relationship. Members of the interdisciplinary team share information willingly in an environment where mutual respect and trust are a norm. ${ }^{[23]}$

\subsubsection{Motivation}

Ballard (2010) considers motivation as one of the factors that affect collaboration success among members of a team. Motivation can result from the individual or the organization. ${ }^{[5,16,17,19,20,33]}$ Intrinsic motivation results in the individual member of the interdisciplinary team always willing to volunteer to work in the context of SG. Organizational leaders and managers can provide a reward to effective team members which will in turn motivate them towards working collaboratively to improve the outcomes of the team. Motivation among interdisciplinary teams can be demonstrated by the willingness of the team members to prioritize shared goals over personal interests. ${ }^{[5,8,16,17,19]}$ Members of an interdisciplinary team will show different levels of commitment depending on their levels of motivation. ${ }^{[19]}$

Stereotypes and perspectives nurtured in different disciplines may influence individual motivation and hence impact their motivation towards the team. ${ }^{[8]}$ Coster et al. ${ }^{[24]}$ considered the commitment to excellence as an important collaboration driver. Giving rewards in the form of time compensation for instance to members of the team can generate pride which improves motivation among team members. ${ }^{[14,16]}$

\subsubsection{Team functional skills}

Members of an interdisciplinary team have a variety of functional skills that enhance SG. ${ }^{[19]}$ They have different skills that will have an impact on the collective decisionmaking of an interdisciplinary team. ${ }^{[8,12,17,28]}$ Lack of skills among groups is likely to hinder effective decisionmaking. ${ }^{[9,32]}$ Each team member contributes skills that help the SG decision-making process. ${ }^{[27]}$ Poor communication skills are a hindrance to collaboration within an interdisci- 
plinary team. ${ }^{[26]}$ Team members can enhance their skills through collaborative training. ${ }^{[8,13,20,21,24,27,33,34]}$ Enhanced collaborative skills in SG may be acquired through training that includes communication, negotiation, and conflict resolution skills. ${ }^{[6,8,16,17,21]}$ Interdisciplinary team members learn to work collaboratively in the SG decision-making process. $^{[6,15,18]}$

\subsection{Organizational factors}

The literature review identified several factors at the level of the organization that have an impact on the SG process. The factors identified include organizational structure, organizational culture, leadership, education, resources, professional boundary and traditional roles, and role ambiguity.

\subsubsection{Organizational structures}

Organizational structures have an impact on the working environment and influence the ways decisions are shared by members of an interdisciplinary team..$^{[5,11-13,19,25,33-35]}$ A flat (non-hierarchal) organizational structures create a good environment that enhances effective SG between nurses and other healthcare providers. ${ }^{[12]}$ Information is easily shared and communication encouraged in flat organizational structures which are important facilitators for collaboration. ${ }^{[12,18,20,27]}$ A rigid bureaucratic organizational structure hinders effective sharing of information among interdisciplinary team members. A rigid bureaucratic organizational structure as well is ineffective in encouraging SG in decision making. ${ }^{[12]}$ The process of top-down information flow takes much time which ultimately hinders effective decision making by the team. ${ }^{[19,27,28]}$

\subsubsection{Organizational culture}

The organizational culture, which refers to the ways of doing things within an organization ${ }^{[23,28,34]}$ is transmitted and evidenced in an organization through the use of language, materials, stories, rituals and symbols. ${ }^{[34]}$ Members of different disciplines work in organizations that have embraced different cultures. When members of an interdisciplinary team come together in an organizational culture that rewards collaborative behavior, the SG process is readily enhanced. Team members and members of different disciplines are likely to collaborate in a climate that encourages collaboration. Members' ways of communication and collaboration are a reflection of the organization's culture. ${ }^{[36]}$ The executive team or management has a central role to play in the development of a positive climate that facilitates communication and shared governance. ${ }^{[37]}$ Members of an interdisciplinary team should be familiar with the change patterns that are likely to affect the culture of the organization. ${ }^{[21,28]}$ Culture change in the process of implementing SG among members of an interdisciplinary team is important for proper communication practices. ${ }^{[16,17,38,39]}$ Hence, the organizational culture affects the attitudes of team members towards members of other disciplines and which in turn enhances collaboration which is an essential element of successful implementation of IDSG.

\subsubsection{Leadership support}

Leadership support impacts the efficiency of engagement in the interdisciplinary SG process. ${ }^{[11,12,25,34]}$ Through supportive leadership it is possible for the team to collaboratively share ideas aimed at improving the quality of healthcare delivered. Reduced leadership support and facilitation of the team is likely to lead to reduced teamwork among the interdisciplinary team members. ${ }^{[8]}$ The environment is not conducive for team members to share when they receive little or no leadership support. The members are less motivated to contribute towards high performance in areas with little or no leadership support. Clear leadership is key to setting up goals that all members of the team will strive to achieve in the SG process. ${ }^{[20]}$ George et al. ${ }^{[25]}$ argued that leaders should have the capacity to skillfully coordinate and sustain the SG process among interdisciplinary team members. Models help nurse managers and leaders in advancing collaborative practices. $^{[12,15]}$

The transformational leadership model empowers team members to innovatively design ideas that are aimed at improving the care process. ${ }^{[33,39]}$ The ideas generated when using transformational leadership help in finding effective solutions to problems in the SG process. ${ }^{[28]}$ Development among team members is enhanced through training by leaders ${ }^{[13,27]}$ which makes it easy for members of different disciplines to collaborate.

Organizational or administrative support is paramount for the success of the SG process. ${ }^{[40]}$ Chief Nurse Officers (CNO) and Chief Medical Officers (CMO) for instance can provide administrative support for nurses and physicians respectively which will help to improve the coordination among interdisciplinary team members. The importance of administrative support in guiding collaboration is emphasized by a large number of studies and scholars. ${ }^{[5-7,11-13,15,21,24-26,28,30,33,41]}$ Some leaders may however not be ready to cede control to members of the interdisciplinary team which negatively affects the SG process. ${ }^{[27,42]}$

\subsubsection{Education}

The education levels of interdisciplinary members affect their abilities to collaborate effectively through communication. ${ }^{[6,7,11,12,19,42]}$ Health workers helping to improve health care quality need training on how they can create and work effectively in teams. The skills they acquire through educa- 
tion will benefit the teams as all members can objectively identify and find solutions to gaps in the collaborative process. Members that are educated on the importance of teams and how they can make teams function efficiently contribute effectively in the collaborative process. Very few members, however, have the capacity or skills to collaborate effectively. ${ }^{[41]}$ Ways of collaborating in a professional manner should be included in training curriculums. ${ }^{[43,44]}$ Inclusion in the curriculum makes it easy for students to learn how they are likely to work together to provide a high quality of service. It also creates a common platform to understand how to work effectively and share ideas in the process of healthcare delivery. ${ }^{[20,42]}$ Education aids in understanding and respecting decisions made by every member through IDSG. Education prepares the team for the change process and makes them ready to deal with challenges that will arise in the process of SG. ${ }^{[11]}$ Anderson et al. ${ }^{[11]}$ argued that education prepares interdisciplinary members to take their roles effectively. Members will acquire skills in the learning process that facilitate the decision-making processes aimed at improving the quality of healthcare.

A nursing curriculum partly trains nurses on ways they can work with other individuals in promoting care. ${ }^{[7]}$ Individuals from other healthcare disciplines are trained and understand the importance of SG in the decision-making process. Early education makes nurses and other healthcare providers aware of the importance to work collaboratively for a common goal, patient care. Such education is important to ensure patient care quality and safety. All professionals should receive an education and training in the best ways to collaborate with individuals from other disciplines in the process of providing care. ${ }^{[42]}$ Pullon ${ }^{[12]}$ argued on the importance of continuous education among nurses and doctors for professional development and effective interdisciplinary collaboration. The education prepares nurses to work with other team members in different healthcare settings. ${ }^{[26]}$ Preparation through education makes it possible for nurses to learn about best practice methods. Nurses can share the information with individuals from other disciplines with justification. A trained and educated nurse is motivated to argue confidently and objectively when placed within the team. ${ }^{[33]}$ Educated nurses are also able to create new strategies for communicating more effectively when placed within a team.

A recent development in healthcare is the concept of interprofessional education and training as a vehicle for preparing healthcare professionals to work together collaboratively for an improved health system and health outcomes. The World Health Organization defines interprofessional education as "interprofessional education occurs when two or more learn about, from, and with each other to enable effective collaboration and improve health outcomes" (p.13). ${ }^{[40]}$ Thus, for successful implementation of IDSG, it is essential that members of health disciplines are educated and trained together on the principles of SG and the factors that influence it.

\subsubsection{Resources}

Resources help in facilitating collaboration and coordination in shared decision-making processes. ${ }^{[21]}$ They include time skills and tools that members of the interdisciplinary team or managers will use to make SG successful in providing care ${ }^{[6,27]}$ Leaders use resources to facilitate training of nurses and other healthcare providers among interdisciplinary team members which eventually makes interdisciplinary members effective members of a team. ${ }^{[19]}$ The collaborative work that interdisciplinary members perform rely on available resources for success. Financial resources, for instance, are important in organizing meetings and enabling staff to attend planned meetings. ${ }^{[44]}$ Knowledge and skills among interdisciplinary team members are important resources that facilitate the collaboration. ${ }^{[7,8,13,19,21,29]}$ Inadequate time limits the development of training programs aimed at improving the efficiency of SG. ${ }^{[5,15,16,24,27,32,42]}$ Jacob et al. ${ }^{[16]}$ argued that motivators and enablers such as financial compensation or compensatory time off to professionals to attend council meetings are important in improving shared governance and collaboration. Lack of sufficient resources may create little or no room for meetings aimed at improving the collaborative process..$^{[5,8,12,15,24,27]}$ Very little time may be available to allow the interdisciplinary team time for meetings which will lead to little sharing and bonding among the team members. ${ }^{[16,19]}$

The times that nurses and members from other disciplines spend in meetings may interfere with normal schedules and ultimately reduces the quality of care due to reduced availability to provide care and lower commitment towards their work. ${ }^{[28]}$ The healthcare environment is busy and very little time can be spared for the IDSG process meetings. ${ }^{[8]}$ Nurses have a lot of work that makes it challenging to devote much time in the SG process. ${ }^{[18]}$ Having more staff to deal with regular duties is a prerequisite to successfully reducing workload and ensuring success when organizing meetings. ${ }^{[5]}$ More dedicated time creates more opportunities to share ideas in the SG process. Reducing workload is key to successful collaboration in the process of enhancing SG among members of an interdisciplinary team. ${ }^{[11,34]}$ Nursing shortage leads to high workloads which adversely affect availability to be involved in interdisciplinary teams. Nurses take much of their time to deal with issues that affect patients and hence become less committed to the SG process. ${ }^{[36]}$ 


\section{Conclusion}

All health care professionals have a shared goal which is to deliver the best care with minimum risks to patient safety. Collaborative interdisciplinary care has been shown to result in better outcomes for patients. The new trend in healthcare is moving to a more innovative way of delivering care that is interdisciplinary SG. The IDSG requires that groups in different disciplines make informed decisions pertaining their work environment as well as those towards patient's care.

\section{Conflicts of Interest Disclosure}

The authors declare that there is no conflict of interest.

\section{REFERENCES}

[1] Porter-O'Grady T. Interdisciplinary shared governance: Integrating practice, transforming health care. Sudbury, MA: Jones \& Bartlett Publishers; 2009.

[2] Swihart D, Hess RG. Shared governance: A Practical approach to transform professional nursing practice. Danvers, MA: HCPro. 2011.

[3] American Nursing Credentialing Center. American nursing credentialing center. 2016 Available from: http://www. nursecredent ialing.org/

[4] Baker JD. The purpose, process, and methods of writing a literature review. Association of Perioperative Registered Nurses Journal. 2016; 103(3): 265-269. PMid:26924364 https ://doi .org/10.1016/j. aorn.2016.01.016

[5] Bennett LM, Gadlin H. Collaboration and Team Science: From Theory to Practice. Journal of Investigative Medicine. 2012; 60(5): 768775. PMid:22525233 https://doi.org/10.2310/JIM. 0b013e $318250871 \mathrm{~d}$

[6] Cadmus E, Helms P, Christopher MA, et al. Interprofessional shared governance: The VNSNY experience. Nursing Management. 2015; 46(9): 34-41. PMid:26295601 https ://doi .org/10.1097/01.N UMA. $0000470773.94859 .2 \mathrm{~d}$

[7] King K, Christo J, Fletcher J, et al. The sustainability of an Australian initiative designed to improve interdisciplinary collaboration in mental health care. International Journal of Mental Health Systems. 2013. PMid:23510105 https ://doi .org/10.1186/1752-4458-7-10

[8] Porter-O'Grady T. Sharing Governance: A Pathway to Interdisciplinary Excellence. Creative Nursing. 2012; 18(4): 150. PMid:23513425 https ://doi.org/10.1891/1078-4535.18.4 .150

[9] O'May F, Buchan J. Shared governance: a literature review. International Journal of Nursing Studies. 1999; 36(4): 281-300. https : //doi.org/10.1016/S0020-7489(99)00023-1

[10] Mickan S, Hoffman SJ, Nasmith L. Collaborative practice in a global health context: Common themes from developed and developing countries. Journal of Interprofessional Care. 2010; 24(5): 492-502. PMid:20718595 https ://doi.org/10.3109/13561821003676 325

[11] Anderson DA, Bankston K, Stindt JL, et al. Interdisciplinary shared governance: A partnership model for high performance in a managed care environment. Seminars for Nurse Managers. 2000; 8(3): 158-169. Available from: https://www.researchgate.net/jou rnal/1066-3851_Seminars_for_nurse_manage

[12] Pullon S. Competence, respect and trust: Key features of successful interprofessional nurse-doctor relationships. Journal of Interprofessional Care. 2008; 22(2): 133-147. PMid:18320449 https: //doi.org/10.1080/13561820701795069

[13] Irajpour A, Alavi M, Abdoli S, et al. Challenges of interprofessional collaboration in Iranian mental health services: A qualitative investigation. Iranian Journal of Nursing And Midwifery Research. 2012;
17(2): 171-177. Available from: http://www. journalonweb.c om/ijnmr/

[14] Ponte PR, Gross AH, Winer E, et al. Implementation an interdisciplinary governance model in a comprehensive cancer center. Oncology Nursing Forum. 2010; 34(3): 611-616. PMid:17573318 https://doi.org/10.1188/07.0NF.611-616

[15] Evan K, Aubry K, Hawkins M, et al. Whole systems shared governance: a model for the integrated health system. Journal of Nursing Administration. 1995; 25(5): 18-27. Available from: https: //www.ncbi.nlm.nih.gov/pubmed/7730930

[16] Jacob A, Roe D, Merrigan R, et al. The Casey Allied Health Model of Interdisciplinary Care (CAHMIC): Development and implementation. International Journal of Therapy \& Rehabilitation. 2013; 20(8): 387 395. Available from: http://www . magonlinelibrary.com/toc /ijtr/current https://doi.org/10.12968/ijtr.2013.20. 8.387

[17] André B, Nøst TH, Frigstad SA, et al. Differences in communication within the nursing group and with members of other professions at a hospital unit. Journal of Clinical Nursing. 2016.

[18] Fewster-Thuente L, Velsor-Friedrich B. Interdisciplinary collaboration for healthcare professionals. Nursing Administration Quarterly. 2008; 32(1): 40-48. PMid:18160862 https://doi.org/10.109 7/01.NAQ.0000305946.31193.61

[19] Kramer M, Schmalenberg C, Maguire P, et al. Structures and practices enabling staff nurses to control their practice. Western Journal of Nursing Research. 2008. PMid:18195080 https ://doi .org/10 $.1177 / 0193945907310559$

[20] Powers S, Bacon CT. Interdisciplinary Shared Governance in Ambulatory Care: One Health System's Journey. Nursing Administration Quarterly. 2016; 40(3): 262-268. PMid:27259130 https: //doi.org/10.1097/NAQ.0000000000000170

[21] Petri L. Concept analysis of interdisciplinary collaboration. Nursing Forum. 2010; 45(2): 73-82. PMid:20536755 https://doi.org/ $10.1111 / j .1744-6198.2010 .00167 . x$

[22] Brewton C, Eppling J, Hobley M. Our voice: An interdisciplinary approach to shared governance. Hospital Topics. 2012; 90(2): 3946. PMid:22671434 https : //doi .org/10.1080/00185868. 201 2.679910

[23] Redman RW. The challenge of interdisciplinary teams. Research and Theory for Nursing Practice. 2006; 20(2): 105-107. Available from: http://www.springerpub.com/research-and-t heory-for-nursing-practice.html PMid:16758714 https: //doi.org/10.1891/rtnp.20.2.105

[24] Coster S, Norman I, Murrells T, et al. Interprofessional attitudes amongst undergraduate students in the health professions: A longitudinal questionnaire survey. International Journal of Nursing Studies. 2008; 45(11): 1667-1681. PMid:18423644 https ://doi.org/10 $.1016 / j . i j n u r s t u .2008 .02 .008$ 
[25] George V, Luhrsen M. Professional Governance: To Act-Not Just Inform. Nurse Leader. 2014; 12(2): 48-54. https : //doi .org/10 $.1016 / j . m n l .2014 .01 .008$

[26] Martin-Rodriguez L, Beaulieu M, D'Armour D, et al. The determinants of successful collaboration: A review of theoretical and empirical studies. Journal of Interprofessional Care. 2005; 1: 132-147. Available from: http://www.tandfonline.com/loi/ijic20

[27] Gilbert JH, Yan J, Hoffman SJ. A WHO report: Framework for action on interprofessional education and collaborative practice. Journal of Allied Health. 2010; 39(3): 196-197. Available from: http://www.who.int/hrh/resources/framework_ac tion/en/ PMid:21174039

[28] Scott L, Caress AL. Shared governance and shared leadership: meeting the challenges of implementation. Journal of Nursing Management. 2005; 13(1): 4-12. PMid:15613089 https://doi .org/10.1 111/j.1365-2834.2004.00455.x

[29] Yeager S. Interdisciplinary collaboration: the heart and soul of health care. Critical Care Nursing Clinics of North America. 2005; 17(2): 143-148. PMid:15862736 https : //doi .org/10.1016/j.ccell. 2005.01 .003

[30] Golanowski M, Beaudry D, Kurz L, et al. Interdisciplinary Shared Decision-Making: Taking Shared Governance to the Next Level. Nursing Administration Quarterly.2007; 31(4): 341-353. PMid:17909433 https://doi.org/10.1097/01.NAQ.0000290 $431.72184 .5 \mathrm{a}$

[31] Newman KP. Transforming organizational culture through nursing shared governance. Nursing Clinics of North America. 2011; 46(1): 45-58. PMid:21320660 https://doi.org/10.1016/j.cnur.2 010.10 .002

[32] Mahdizadeh M, Heydari A, Moonaghi HK. Clinical Interdisciplinary Collaboration Models and Frameworks from Similarities to Differences: A Systematic Review. Global Journal Of Health Science. 2015; 7(6): 170. PMid:26153158 https ://doi.org/10.5539/gj hs. v7n6p170

[33] Hall P. Interprofessional teamwork: Professional cultures as barriers. Journal of Interprofessional Care. 2005; 19(sup1): 188-196.

[34] Lockhart-Wood K. Collaboration between nurses and doctors in clinical practice. British Journal of Nursing. 2000; 9(5): 276-280.
PMid:11042783 https://doi.org/10.12968/bjon.2000.9.5 .6363

[35] Leggat SG. Effective healthcare teams require effective team members: defining teamwork competencies. BMC Health Services Research. 2007; 7(1): 1-10. PMid:17284324 https://doi.org/10 .1186/1472-6963-7-17

[36] Hoffman SJ, Harnish D. The merit of mandatory interprofessional education for pre-health professional students. Medical Teacher. 2007; 29(8): e235-e242. PMid:18236267 https ://doi.org/10.1080/ 01421590701551672

[37] Jansen L. Collaborative and interdisciplinary health care teams: ready or not? Journal of Nursing Administration. 2008; 40(10): 411-416. https://doi.org/10.1016/j.profnurs. 2007.06 .013

[38] Hoying C, Allen SR. Enhancing shared governance for interdisciplinary practice. Nursing Administration Quarterly. 2011; 35(3): 252259. PMid:21654486 https://doi.org/10.1097/NAQ.0b013e 3181ff3a1d

[39] Dietrich SL, Kornet TM, Lawson DR, et al. Collaboration to partnerships. Nursing Administration Quarterly. 2010; 34(1): 49-55. PMid:20023561 https://doi.org/10.1097/NAQ.0b013e3181 c95ea7

[40] Would Health Organization. Health systems. Available from: http://www.who.int/topics/health_systems/qa/en/0r ganization

[41] Ballard N. Factors associated with success and breakdown of shared governance. Journal of Nursing Administration. 2010; 40(10): 411416. PMid:20859091 https://doi.org/10.1097/NNA . Ob013e $3181 \mathrm{f} 2 \mathrm{eb} 14$

[42] Canadian Interprofessional Health collaborative. Interprofessional Education \& Core Competencies. 2007.

[43] Edwards C. Using interdisciplinary shared governance and patient rounds to increase patient safety. Medsurg Nursing. 2008; 17(4): 255. PMid:18807861

[44] Erickson JI, Hamilton GA, Jones DE, et al. The value of collaborative governance/staff empowerment. Journal of Nursing Administration. 2003; 33(2): 96-104. Available from: http://citeseerx.ist.psu.edu/viewdoc/download ?doi=10.1.1.477.890\&rep=rep1\&type=pdf 